\title{
FABRICATION OF A BIOMIMETIC CORRUGATED POLYSILICON DIAPHRAGM WITH ATTACHED SINGLE CRYSTAL SILICON PROOF MASSES
}

\author{
Kyutae Yoo, J.-L. A. Yeh, N. C. Tien*; C. Gibbons**, Q. Su**, W. Cui**, and R. N. Miles**, \\ School of Electrical and Computer Engineering, Cornell University, \\ Ithaca, NY 14853, USA, yoo@ee.cornell.edu \\ *Department of Electrical and Computer Engineering, University of California, Davis CA 95616 \\ **Department of Mechanical Engineering, SUNY, P.O. 6000 Binghamton, NY 13902-6000
}

\section{SUMMARY}

This paper reports a new approach to the fabrication of 3D structured diaphragms using integrated surface and deep reactive ion etching (DRIE) bulk silicon micromachining on a silicon-on-insulator (SOI) wafer. The fabrication process has been applied to a $1 \mathrm{~mm} \times 2$ $\mathrm{mm} \times 1.2 \mu \mathrm{m}$ diaphragm designed for a biomimetic directional microphone. The membrane has two $200 \mu \mathrm{m}$ $\times 380 \mu \mathrm{m} \times 20 \mu \mathrm{m}$ silicon proof masses, solid stiffeners, and a $20 \mu \mathrm{m}$ deep corrugation. Acoustic measurements of the diaphragm using laser vibrometry have demonstrated high directional sensitivity of the device.

Keywords: Integrated surface and DRIE bulk silicon micromachining, biomimetic device, and diaphragm

\section{Introduction}

Non-planar micromachined diaphragms that make use of corrugations and bosses have become key components in various micromechanical devices such as microphones, microvalves, micropumps, and accelerometers. As new application areas are discovered and specialized devices are designed, more complex diaphragms will be required. However, two well-established methods for fabricating 3D structured diaphragms have certain drawbacks limiting the design flexibility. In the electrochemical etch-stop technique, which combines anodic passivation of silicon with a reverse bias of $\mathrm{p}-\mathrm{n}$ junction, n-type silicon is formed either by epitaxial growth or ion implantation and diffusion on a p-type wafer. It is impractical to construct "proof mass" types of 3D structures on silicon membranes with epitaxially grown n-type layers due to the uniform growth of the epi-layer, whereas it is attainable with ion implanted and diffused n-type layer by double diffusion [1]. The second method utilizes heavily boron-doped $\left(\mathrm{p}^{++}\right)$layers as etch stop-layers. In most cases, they are ion implanted and diffused.
However, these layers can also be epitaxially grown. The stress induced by the high dopant concentration can considerably alter critical mechanical factors such as the resonant frequency of the diaphragm. In addition, the substantial surface damage from implantation precludes the formation of active electronic devices. In both methods, which employ etch-stop layers created by ion implantation and diffusion, the achievable thickness of the structures on diaphragms is upper-bounded by the maximum dopants diffusion depth (on the order of $15 \mu \mathrm{m}$ ) [2].

Our method integrates surface micromachining with bulk silicon micromachining to fabricate $3 \mathrm{D}$ structured membranes [3]. It utilizes a buried oxide layer in a SOI wafer as an etch-stop layer. DRIE allows precision patterning of single crystal silicon structures such as proof masses, and stiffeners. The thicknesses of the structures and the depths of corrugations are determined by the thickness of the device layer on the SOI wafer. Silicon is etched from the backside of the wafer using DRIE unlike the conventional methods, which use anisotropic etchants (EDP, KOH, TMAH).

To demonstrate the potential of our new approach for the fabrication of 3D structured diaphragms, in this work, a biomimetic corrugated polysilicon diaphragm with single crystal silicon proof masses and stiffeners for a directional microphone is presented.

\section{Design}

Preliminary studies on the parasitoid fly Ormia ochracea reveal that the fly's ears are mechanically coupled by a flexible bridge, which is utilized to achieve directionality [4]. The mechanical link between ears provides two resonant modes, a rocking mode and an in-phase mode. Linear combinations of these two modes produce asymmetrical mechanical responses of the tympana, thereby generating 


\section{TRANSDUCERS '01 EUROSENSORS XV}

The 11th International Conference on Solid-State Sensors and Actuators, Munich, Germany, June 10 - 14, 2001 p.130-133

mechanical interaural delays and amplitude differences resulting in directionality. Our diaphragm design, shown in figure 1 is based on the mechanical model of the fly's ear [5]. The $1 \mathrm{~mm} \times 2 \mathrm{~mm} \times 1.2 \mu \mathrm{m}$ diaphragm is constructed of polysilicon. It has two 200 x 380 x $20 \mu \mathrm{m}^{3}$ single crystal silicon proof masses and solid stiffeners in order to create two fundamental vibration modes. The diaphragm makes use of a 20 $\mu \mathrm{m}$-deep corrugation to relieve in-plane stress and increase mechanical sensitivity. It is rounded at the corners to reduce stress concentration.

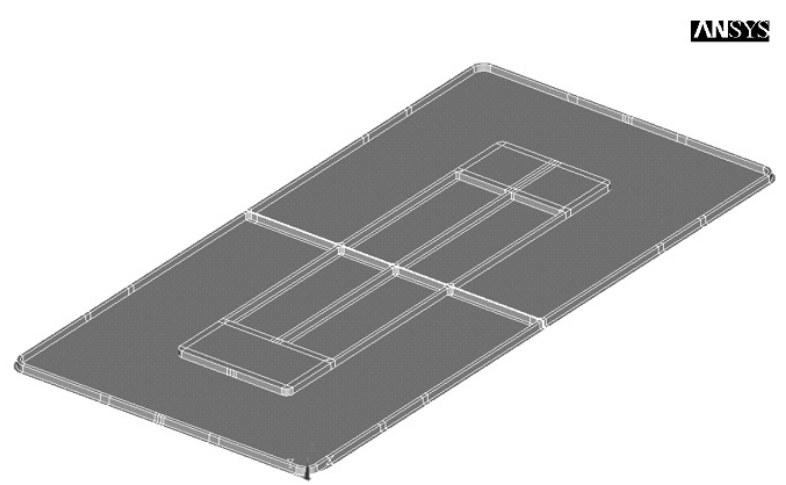

Fig. 1: Diaphragm model.

\section{Fabrication}

Biomimetic diaphragms for directional microphones have been fabricated on 4-inch silicon-on-insulator (SOI) substrates using integrated surface and deep reactive ion etching (DRIE) bulk silicon micromachining.

\section{Oxide Block Formation}

The proof masses and corrugations for the diaphragms are constructed by transforming unwanted bulk silicon into oxide blocks and removing them at the releasing step of the process. These oxide blocks are created by oxidation of closely spaced silicon beams followed by LTO oxide deposition and chemical mechanical polishing (CMP). Earlier experiments showed a large compressive stress induced by the volume expansion from oxidation and the coefficients of thermal expansion (CTEs) mismatch between silicon and $\mathrm{SiO}_{2}$ caused a severe bending of the $1-\mu \mathrm{m}$-wide silicon beams after oxidation [3]. Highly stressed sections of the beams experienced retardation of oxidation, and incompletely oxidized silicon residues, which could prevent the successful completion of the devices. In our new silicon beam design, sets of $25 \sim 50-\mu \mathrm{m}$-long, and $1-\mu \mathrm{m}$-wide beams are separated by $2 \mu \mathrm{m}$ from each other to allow the expansion of oxide, and supported by lateral beams to increase structural rigidity of oxide blocks for the following processes. In figure 2 , only a slight bending of beams is observed after oxidation with the new design, leading to the complete oxidation of the beams. Also, the unchanged width of the gaps between the beams $(2 \pm 0.2 \mu \mathrm{m})$ after oxidation makes it possible to fill and seal them with less LTO oxide.

a)

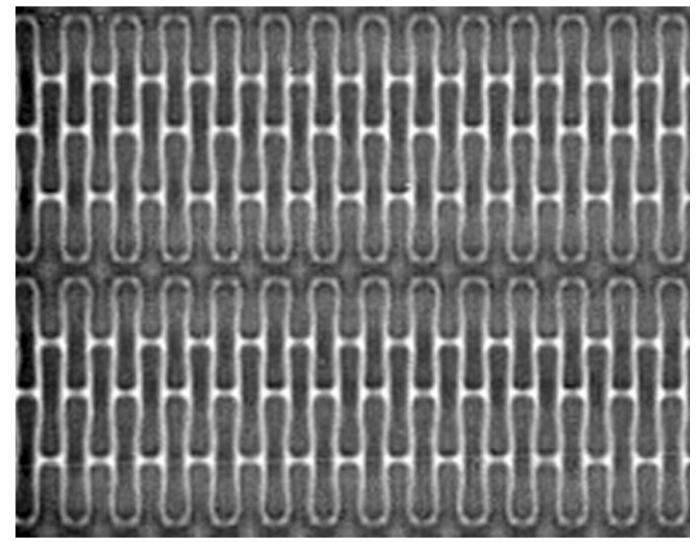

b)

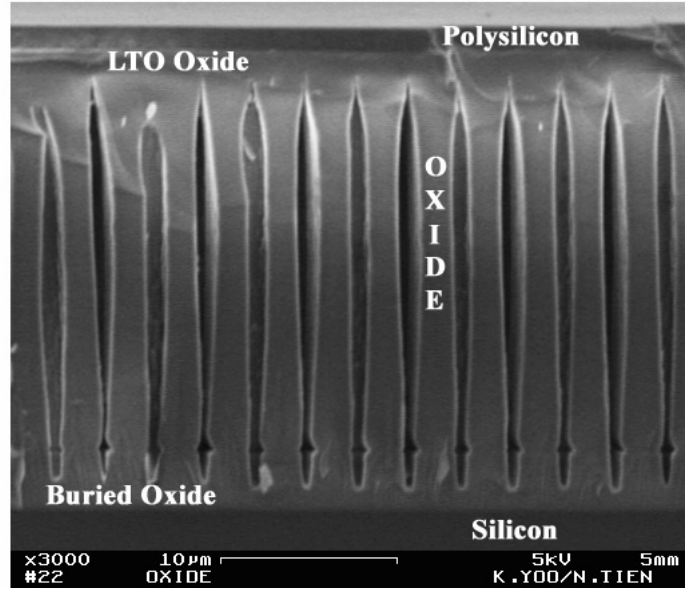

Fig. 2: a) Beams after oxidation, b) Cross-sectional SEM picture of Oxide Block with 1.2 $\mu$ m polysilicon

\section{Process Flow}

The process is depicted schematically in figure 3. Our starting material is a SOI wafer with $20-\mu$ m-thick device silicon and $2-\mu \mathrm{m}$-thick buried oxide. $20-\mu \mathrm{m}$ deep trenches are etched using DRIE (fig. 3a). The silicon beams are thermally oxidized. LTO (Low Temperature Oxide) is deposited to fill and seal the gaps between oxidized beams. Once the unwanted silicon is transformed into the oxide blocks, the surface is planarized by chemical-mechanical polishing (CMP) to allow the subsequent surface micromachining (fig. 


\section{TRANSDUCERS '01 EUROSENSORS XV}

The 11th International Conference on Solid-State Sensors and Actuators, Munich, Germany, June 10 - 14, 2001 p.130-133

3b). Silicon is etched using DRIE for corrugations (fig. $3 \mathrm{c})$. 1.2- $\mu \mathrm{m}$-thick phosphorus doped polysilicon is deposited at $580^{\circ} \mathrm{C}$ and annealed at $1150^{\circ} \mathrm{C}$ for $80 \mathrm{~min}$ in a dry $\mathrm{N}_{2}$ ambient to provide slight tensile stress (fig. 3d). Silicon is removed from the backside of the wafer using DRIE, and the etch stops at the buried oxide layer (fig. 3e). Using a hydrofluoric acid solution, the oxide blocks are removed, and the corrugated diaphragms with proof masses are released (fig. 3f). Figure 4 shows the fabricated diaphragm.

a)

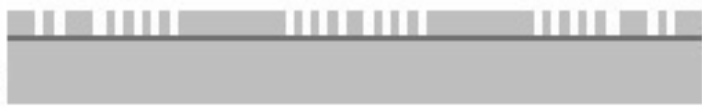

b)

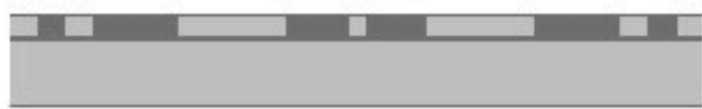

c)

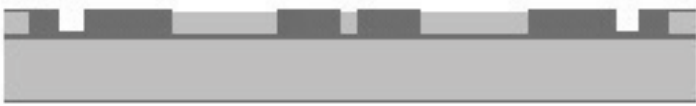

d)

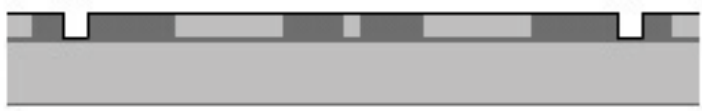

e)

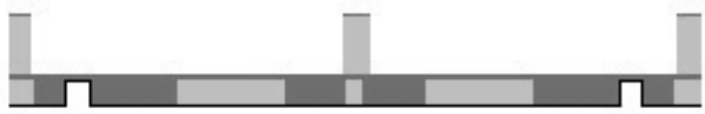

f)

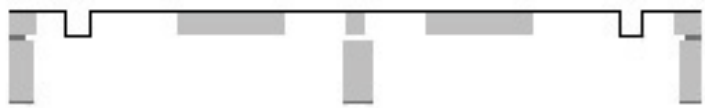

Fig. 3: Process sequence for the biomimetic corrugated polysilicon diaphragm with single crystal silicon proof masses.

\section{Results}

Laser vibrometry has been employed to acquire measurements of the sound induced vibrations of the diaphragm. The testing set-up utilizes a Polytec laser vibrometer (OFV 302 optical-head and OFV-2100 electronics unit), a loudspeaker, and a reference microphone (B\&K 4138). The displacements at 25 locations on the diaphragm were measured as a function of frequency. The incident sound waves were generated by a loudspeaker positioned at $45^{\circ}$ angle relative to the longitudinal axis of the diaphragm. In figure 5, the deflection shapes of the diaphragm for three different frequencies are shown. At the first resonant frequency $(\sim 16 \mathrm{kHz})$, the diaphragm rocks about the pivot point (fig. 5a). On the other hand, at the second resonant frequency $(\sim 25 \mathrm{kHz})$, both sides of the diaphragm move with the same amplitude and phase (fig. 5b).

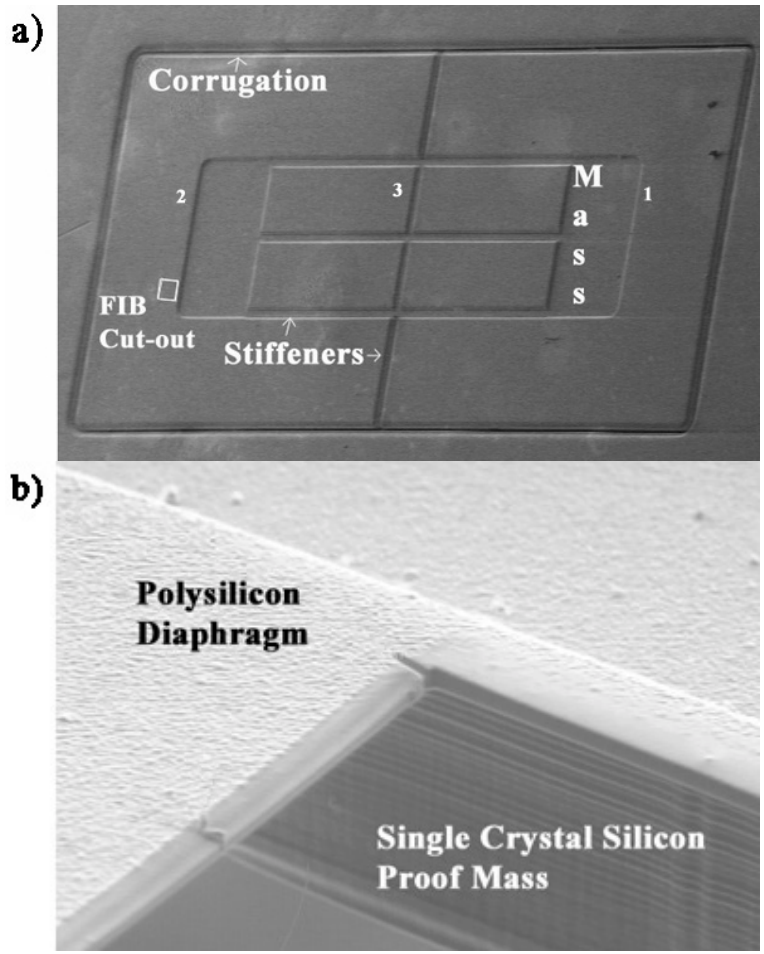

Fig. 4: SEM of the diaphragm: a) top view, b) side view of proof mass attached to diaphragm through window cut by FIB (dotted area in a).

Frequencies between the first and second resonant frequencies result in the linear combination of these two modes producing asymmetric shapes of the diaphragm with a significant difference in amplitudes, thus providing directionality (fig. 5c). The results show that the diaphragm has very similar mode shapes when responding to sound pressure and directional sensitivity compared to the fly's ears.

\section{Conclusion}

The new approach to the fabrication of non-planar diaphragms using integrated surface and deep reactive ion etching (DRIE) bulk silicon micromachining on a silicon-on-insulator (SOI) wafer offers vital advantages of excellent precision in dimensions of the structures, and high flexibility in designs over conventional etchstop techniques. Our method makes use of DRIE which gives high-aspect-ratio up to 30:1 (sidewall angles $90 \pm 2^{\circ}$ ) to etch silicon from the backside of the wafer [2]. This enables us to build arrays of closely spaced 


\section{TRANSDUCERS '01 EUROSENSORS XV}

The 11th International Conference on Solid-State Sensors and Actuators, Munich, Germany, June 10 - 14, 2001 p.130-133

diaphragms for various MEMS applications. Furthermore, the variations of dimensions of diaphragms due to the thickness variations of the wafer itself can be minimized.

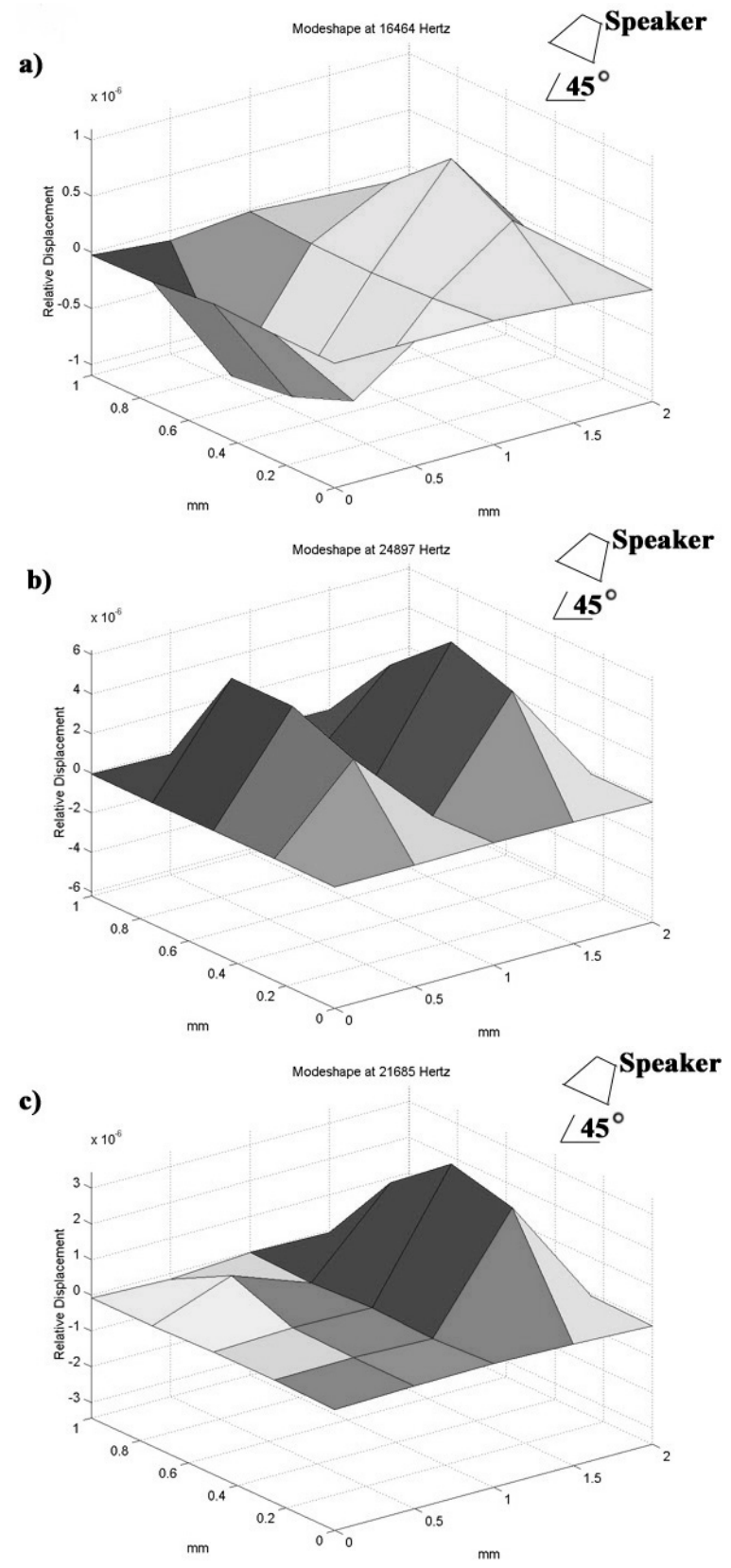

Fig. 5: 3D-plots of deflection shapes of the diaphragm: a) rocking (out-of-phase) mode, b) translational (inphase) mode, c) linear combination of two modes.

Our method has been applied to a diaphragm, which incorporates a corrugation, proof masses, and stiffeners to mimic the ears of the parasitoid fly, Ormia ochracea.
The successfully fabricated diaphragm has two resonant modes of vibration, and the linear combination of these two modes results in directionality.

\section{ACKNOWLEDGMENTS}

The authors would like to thank Steven Herschbein, and Aron Shore of IBM for FIB service, and the staff of the Cornell Nanofabrication Facility. The authors also would like to thank D. Chang, H. Jiang, D.T. McCormick, K.V. Madanagopal, and M. Pai for useful discussions.

\section{REFERENCES}

[1] R. Huster, and A. Stoffel, "Vertically Structured Silicon Membranes by Electrochemical Etching," Sensors and Actuators, vol. A21-A23, pp. 899903, 1990.

[2] G. T. A. Kovacs, Micromachined Transducers sourcebook, McGraw-Hill press, New York, 1998.

[3] J.-L. A. Yeh, H. Jiang, and N. C. Tien, ’Integrated Polysilicon and DRIE Bulk Silicon Micromachining for an Electrostatic Torsional Actuator," J. Microelectromech. Syst., vol. 8, no. 4, pp. 456-465, 1999.

[4] R.N. Miles, D. Robert, and R. R. Hoy, "Mechanically coupled ears for directional hearing in the parasitoid fly Ormia ochracea," J.Acoust. Soc. Am., vol. 98, no. 6, pp. 3059-3070, 1995.

[5] C. Gibbons, and R. N. Miles, "Design of a Biomimetic Directional Microphone Diaphragm," Proceedings of International Mechanical Engineering Congress and Exposition., Orlando FL, Nov. 2000. 